\title{
Surgical Procedure for Sporadic Colorectal Cancer in Patients with Mild Ulcerative Colitis
}

\author{
Motoi Uchino $^{\mathrm{a}}$ Hiroki Ikeuchi $^{\mathrm{a}}$ Hiroki Matsuoka $^{\mathrm{a}}$ \\ Toshihiro Bando $^{a} \quad$ Akihiro Hirata ${ }^{a}$ Satoru Yasukawa ${ }^{c}$ \\ Yoshio Takesue $^{b}$ Naohiro Tomita ${ }^{a}$ \\ ${ }^{a}$ Department of Lower Gastroenterological Surgery and ${ }^{b}$ Division of Infection \\ Control and Prevention, Hyogo College of Medicine, Nishinomiya, and \\ 'Department of Surgical Pathology, Kyoto Prefectural University of Medicine, \\ Kyoto, Japan
}

\section{Key Words}

Ulcerative colitis - Colitic cancer - Sporadic cancer - Restorative proctocolectomy . Partial resection

\begin{abstract}
Restorative proctocolectomy is recognized as the standard procedure for colitic cancer in patients with ulcerative colitis (UC). However, whether this represents the optimal procedure for UC patients with sporadic cancer remains questionable, as functional quality of life differs substantially between patients with proctocolectomy and partial resection. This study considered possible problems associated with sporadic cancer in UC. Case 1 is a 55-year-old man with a 3-year history of UC who was treated with endoscopic resection for sporadic adenocarcinoma in the rectum. Low anterior resection was subsequently performed due to deep invasion. The final diagnosis was pT3. Differentiating between histopathological diagnoses of sporadic and colitic cancer was difficult. Case 2 is a 71-year-old woman with a 6-year history of UC who was diagnosed with type 1 sporadic sigmoid colon cancer. Dementia and umbilical hernia were present as complications. Total colectomy was performed in consideration of the coexisting complications. Although partial resection for sporadic cancer could be favorable in mild colitis, further immunosuppressive treatments have the potential to elevate the risk of recurrence for advanced cancer. Restorative proctocolectomy may be safer to avoid further recurrent colitis and cancer except in elderly patients or those with other complications.
\end{abstract}




\section{Introduction}

The risk of developing colorectal cancer (CRC) is known to be significantly increased in patients with ulcerative colitis (UC). Surveillance colonoscopy has been implemented to identify colitis-associated neoplasms, including dysplasia-associated lesion or mass (DALM) and colitis-associated CRC.

The overall prevalence of CRC in patients with UC has been estimated as $3.7 \%$ in a meta-analysis [1]. Duration of chronic UC $>10$ years and pancolitis are known to represent risk factors for UC-associated CRC (UC-CRC) [2]. On the other hand, sporadic adenoma and adenocarcinoma can arise coincidentally in patients with UC. Furthermore, distinguishing between DALM and sporadic adenoma-like mass (ALM) is crucial, as prophylactic restorative proctocolectomy is usually recommended for DALM, while polypectomy may suffice for ALM. Even in sporadic adenocarcinoma, partial resection may be adequate compared with restorative proctocolectomy in view of anal function and quality of life. We report herein two considerable cases of sporadic adenocarcinoma in patients with chronic UC and consider the associated problems.

\section{Case Reports}

Case 1

A 55-year-old man with a 3-year history of UC was referred to our hospital. He had been examined by colonoscopy every year at the previous outpatient clinic. A diagnosis of UC was made on the basis of initial colonoscopic findings and confirmed by the typical histological features seen in biopsy samples. The patient had left-side colitis and had been treated using aminosalicylate and azathiopurine for refractory disease without corticosteroid administration. After 3 years of asymptomatic UC, a lateral-spreading tumor with a diameter of about $10 \mathrm{~mm}$ in Ra section was detected in the upper rectum (fig. 1). A biopsy specimen had been highly suspicious of well-differentiated tubular adenocarcinoma and endoscopic mucosal resection (EMR) was performed. Histopathological findings after EMR revealed positive results for malignant cells at the vertical margin and invasion into the deeper submucosal layer (fig. 2). Rectal mucosa around the tumor revealed mild inflammatory cell infiltration, crypt abscess and crypt distortion which was compatible with UC. Sporadic rectal cancer was suspected by endoscopic and histopathological findings and diagnosed with post-EMR staging of T2, N0, stage 1. Low anterior resection (LAR) was therefore performed. The resected specimen showed invasion to the subserosal layer with positive p53 immunohistostaining and metastasis into lymph nodes classified as pT3 and pN1, stage 3B (fig. 3 ). Adjuvant chemotherapy was required and performed postoperatively. Although colitis worsened within 1 year after surgery and was diagnosed as mild colitis, the patient was managed favorably using only aminosalicylate.

\section{Case 2}

A 71-year-old woman with a 6-year history of UC was admitted to our hospital complaining of melena. Although she was diagnosed with left-side UC based on endoscopic and histopathological findings on presentation, recovery was seen with treatment comprising only oral aminosalicylate without refractory disease. Type 1 tumor surrounded by non-inflamed mucosa was seen in the sigmoid colon on colonoscopy and diagnosed as well-differentiated adenocarcinoma from biopsy

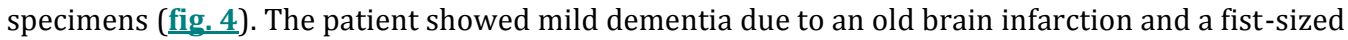
umbilical hernia with prolapsed transverse colon. She was diagnosed with sporadic sigmoid colon cancer and total colectomy and ileorectal anastomosis were performed in consideration of the concomitant complications. The resected specimens showed well-differentiated adenocarcinoma within the mucosal layer, representing pTis tumor without lymph node metastasis (stage 0 ). Mild inflammatory cell infiltration and mild crypt distortion compatible with UC were seen in the left-sided colon and diagnosed as sporadic cancer against a background of mild colitis. 


\section{Discussion}

The pathological differences between DALM and ALM are considered to complicate the acquisition of a proper diagnosis, particularly when based on biopsy specimens [3]. EMR or endoscopic submucosal resection are considered to be good for achieving an accurate diagnosis, particularly in patients with early-stage cancer including also dysplasia, because these lesions are mainly differentiated by combined analysis of histological growth pattern and gross appearance [4]. Safety and efficacy of endoscopic resection have also been evaluated in previous reports [5-7]. From the perspective of clinical considerations, accurate pathological diagnosis is very important for distinguishing between different pathological entities, given the different therapeutic consequences such as endoscopic polypectomy for ALM and potential proctocolectomy for DALM. Similar considerations are also involved in discriminating between sporadic adenocarcinoma and UC-CRC. In patients with mild colitis similar to case 1 in this study, partial resection (but not total proctocolectomy) can be considered if an accurate diagnosis of sporadic cancer is required. EMR or endoscopic submucosal resection may be suitable for such cases, even in lesions with suspected deep invasion to the submucosal layer. However, anal function and quality of life differ substantially between total proctocolectomy with ileal pouch anal anastomosis (IPAA) and LAR. A key point is that further proctocolectomy and IPAA would be difficult after partial resection, particularly after LAR. Proctocolectomy with IPAA may be suitable for sporadic cancers in the lower rectum. In patients with UC, irrespective of the degree of colitis, LAR should not be selected for sporadic cancer in the lower rectum except in older patients, based on considerations of quality of life and risk of further colitis.

On the other hand, among patients with moderate or worse inflammation of colitis that requires aggressive medical treatment, total proctocolectomy with IPAA should be performed even in patients with sporadic cancer, for two main reasons.

First, further IPAA may not be able to be performed after partial resection due to lymphadenectomy or adhesions at the surgical site. If ileocecal resection or right hemicolectomy with lymphadenectomy is performed for advanced sporadic cancer of the right colon, excision of the marginal arcade of the ileocolic artery that supplies the ileal pouch could preclude IPAA, particularly in hand-sewn IPAA with mucosectomy, because the ileal pouch cannot reach the site of anastomosis. The surgical procedure in the pelvic space after surgery for rectal cancer could influence the possibility of IPAA due to adhesions from the initial surgery. However, left hemicolectomy represents an exception to this limitation, because the procedure is not associated with IPAA, as in case 2 from this study.

Second, administration of immunomodulators or biologics for recurrent or refractory colitis may be restricted in patients with advanced cancer after partial resection. Previous studies and guidelines have demonstrated that the risk of new cancer development is not increased with the administration of immunomodulators or biologics [8, 9]. However, infliximab reportedly represents a potential risk for developing previously existing cancer in rheumatoid patients, thiopurine could be a high risk for various previously existing cancer development in transplant patients, and calcineurin inhibitor also could increase the recurrence rate in patients after liver transplantation for hepatocellular carcinoma [10-12]. Although no links in patients with inflammatory bowel disease have yet been confirmed, avoiding administration of 
these agents in patients with advanced cancer requiring adjuvant chemotherapy appears prudent.

In elderly patients with poor anal function, surgical procedures should obviously be considered based on overall considerations including prognosis of the cancer, degree of inflammation with colitis and potential requirements for future treatment.

In case 1, although the initial diagnosis before and shortly after EMR was massive invasion to the submucosal layer, the final histopathological diagnosis was invasion to the subserosal layer. This discrepancy may be due to the easy invasive behavior of cancer cells in UC. Although UC-CRC shows poor prognosis compared to sporadic CRC, histopathological studies have suggested similarities between these cancers [13]. However, UC-CRC tends to show poorer differentiation when invading to submucosal or deeper layers, even if the cancer is well-differentiated in the lamia propria [14]. In a previous study, although the degree of differentiations was similar in both sporadic CRC and UC-CRC, prognosis was poorer in UC-CRC than in sporadic CRC [13]. The earlier invasive characteristics of UC-CRC may be associated not only with the characteristic dysplasia-carcinoma sequence, but also with invasive behavior against an inflamed background of the mucosal and submucosal layers as a feature of colitis, even in sporadic lesions $[4,15]$. Even with sporadic CRC in patients with UC, a tendency toward deeper invasion may be a feature.

\section{Conclusion}

In view of the risk of recurrent colitis and cancer, partial resection might be less advantageous than proctocolectomy. Proctocolectomy with IPAA may be safe for advanced CRC regardless of the origin as colitic or sporadic cancer, because of the difficulty of differentiation, ready invasive behavior against an inflamed background and worsened potential for further progressive colitis in younger patients. Decisions on surgical procedures should be made based on full consideration of background factors including age, degree of colitis and cancer prognosis.

\section{Disclosure Statement}

None of the authors have any conflicts of interest to declare. 


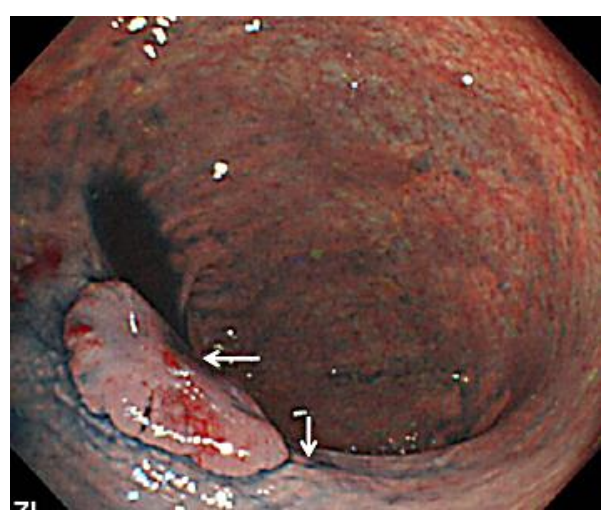

Fig. 1. Endoscopic findings for case 1. Colonoscopic study revealed rectal cancer. A lateral-spreading tumor with clear borders, slightly central depression and a diameter of about $10 \mathrm{~mm}$ was apparent in the upper rectum on colonoscopy $(\longleftarrow)$. The colonic mucosa around the tumor showed mildly inflamed erosion without disappearance of vascular network patterns compatible with mild UC $(\downarrow)$.

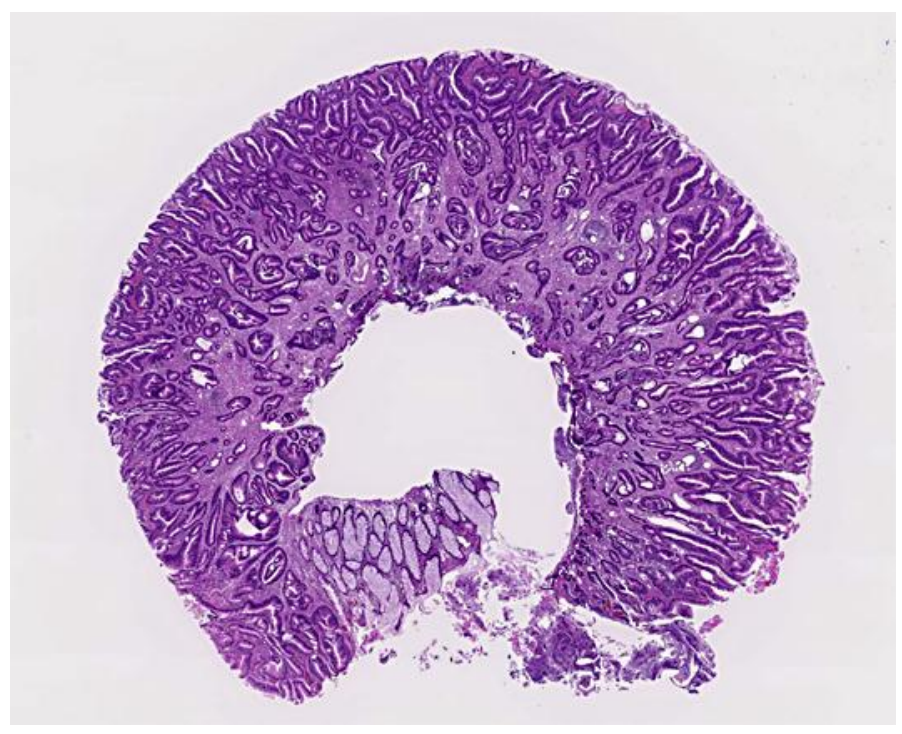

Fig. 2. Histopathological findings for endoscopic resection. Histopathological examination for the endoscopically resected specimen with hematoxylin and eosin staining $(\times 20)$ revealed malignant cells which were apparent in the vertical margins with invasion into the deeper submucosal layer. 


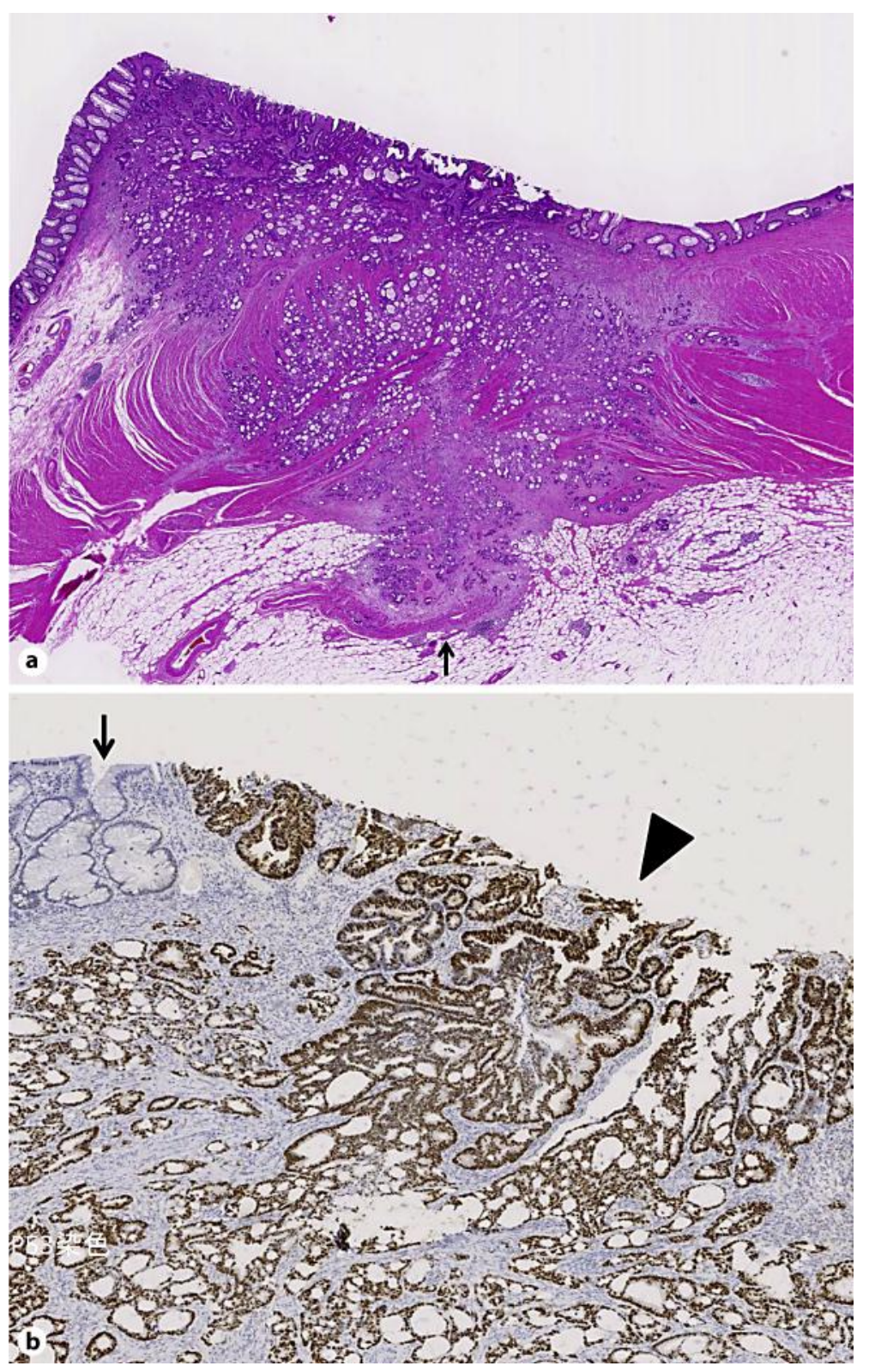

Fig. 3. Histopathological findings for the resected specimen. a Histopathological findings for the resected specimen with hematoxylin and eosin staining $(\times 40)$ after LAR revealed deep invasion to the subserosal layer $(\uparrow)$. b Positive immunohistostaining with anti-p53 antibody $(\times 40)$ was recognized in the whole part of invasive tumor (arrowhead) with negative staining in crypts around the tumor $(\downarrow)$. 


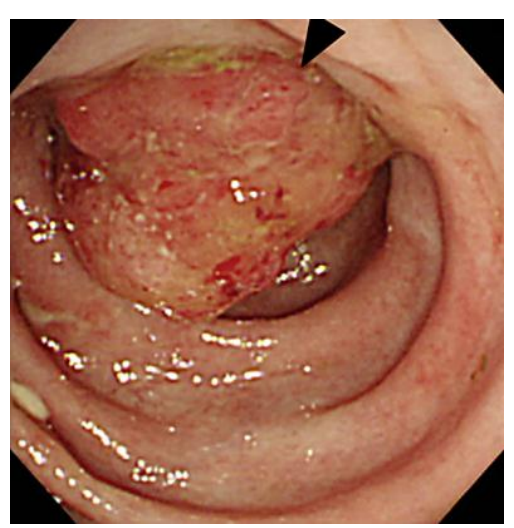

Fig. 4. Endoscopic findings for case 2. Type 1 tumor (arrowhead) with mild erosion and inconspicuous inflammation in the surrounding mucosa was revealed at the sigmoid colon on colonoscopy.

\section{References}

1 Eaden JA, Abrams KR, Mayberry JF: The risk of colorectal cancer in ulcerative colitis: a meta-analysis. Gut 2001;48:526-535.

-2 Ullman T, Odze R, Farraye FA: Diagnosis and management of dysplasia in patients with ulcerative colitis and Crohn's disease of the colon. Inflamm Bowel Dis 2009;15:630-638.

-3 Mueller E, Vieth M, Stolte M, Mueller J: The differentiation of true adenomas from colitis-associated dysplasia in ulcerative colitis: a comparative immunohistochemical study. Hum Pathol 1999;30: 898-905.

4 Tarmin L, Yin J, Harpaz N, Kozam M, Noordzij J, Antonio LB, Jiang HY, Chan O, Cymes K, Meltzer SJ: Adenomatous polyposis coli gene mutations in ulcerative colitis-associated dysplasia and cancers versus sporadic colon neoplasms. Cancer Res 1995;55:2035-2038.

5 Neumann H, Vieth M, Langner C, Neurath MF, Mudter J: Cancer risk in IBD: how to diagnose and how to manage DALM and ALM. World J Gastroenterol 2011;17:3184-3191.

-6 Hurlstone DP, Sanders DS, Atkinson R, Hunter MD, McAlindon ME, Lobo AJ, Cross SS, Thomson M: Endoscopic mucosal resection for flat neoplasia in chronic ulcerative colitis: can we change the endoscopic management paradigm? Gut 2007;56:838-846.

7 Kisiel JB, Loftus EV Jr, Harmsen WS, Zinsmeister AR, Sandborn WJ: Outcome of sporadic adenomas and adenoma-like dysplasia in patients with ulcerative colitis undergoing polypectomy. Inflamm Bowel Dis 2012;18:226-235.

-8 Sandborn WJ, Loftus EV: Balancing the risks and benefits of infliximab in the treatment of inflammatory bowel disease. Gut 2004;53:780-782.

-9 Askling J, Fahrbach K, Nordstrom B, Ross S, Schmid CH, Symmons D: Cancer risk with tumor necrosis factor alpha inhibitors: meta-analysis of randomized controlled trials of adalimumab, etanercept, and infliximab using patient level data. Pharmacoepidemiol Drug Saf 2011;20:119-130.

10 Dixon WG, Watson KD, Lunt M, Mercer LK, Hyrich KL, Symmons DP; British Society for Rheumatology Biologics Register Control Centre Consortium; British Society for Rheumatology Biologics Register: Influence of anti-tumor necrosis factor therapy on cancer incidence in patients with rheumatoid arthritis who had a prior malignancy: results from the British Society for Rheumatology Biologics Register. Arthritis Care Res 2010;62:755-763.

11 Karran P, Attard N: Thiopurines in current medical practice: molecular mechanisms and contributions to therapy-related cancer. Nat Rev Cancer 2008;8:24-36.

12 Vivarelli M, Cucchetti A, La Barba G, Ravaioli M, Del Gaudio M, Lauro A, Grazi GL, Pinna AD: Liver transplantation for hepatocellular carcinoma under calcineurin inhibitors: reassessment of risk factors for tumor recurrence. Ann Surg 2008;248:857-862. 
13 Watanabe T, Konishi T, Kishimoto J, Kotake K, Muto T, Sugihara K: Ulcerative colitis-associated colorectal cancer shows a poorer survival than sporadic colorectal cancer: a nationwide Japanese study. Inflamm Bowel Dis 2011;17:802-808.

14 Mikami T, Yoshida T, Numata Y, Kikuchi M, Araki K, Nakada N, Okayasu I: Invasive behavior of ulcerative colitis-associated carcinoma is related to reduced expression of CD44 extracellular domain: comparison with sporadic colon carcinoma. Diagn Pathol 2011;6:30.

15 Watanabe T, Kobunai T, Toda E, Kanazawa T, Kazama Y, Tanaka J, Tanaka T, Yamamoto Y, Hata K, Kojima T, Yokoyama T, Konishi T, Okayama Y, Sugimoto Y, Oka T, Sasaki S, Ajioka Y, Muto T, Nagawa H: Gene expression signature and the prediction of ulcerative colitis-associated colorectal cancer by DNA microarray. Clin Cancer Res 2007;13:415-420. 